\title{
An der Schnittstelle von Flucht und Behinderung. Ergebnisse einer Analyse der Unterbringungs- und Versorgungssituation von Geflüchteten mit Behinderung in der Kommune München
}

\section{Annette Korntheuer}

\section{Einleitung}

Auch wenn sich die Datenlage am Schnittpunkt von Flucht und Behinderung bislang undifferenziert und lückenhaft darstellt, wird national und international eine Zunahme von Publikationen, anwendungsbezogener Forschung und Praxisinitiativen in den letzten fünf Jahren deutlich (Steiner, od; Otten et al., 2017; AWO Bundesverband e.V., 2017). Dennoch wird die Unsichtbarkeit von Geflüchteten mit Behinderung in den wissenschaftlichen Diskursen immer wieder thematisiert. Emma Pearce (2015) beschreibt, wie Menschen mit Behinderung in Konflikt- und Fluchtsituationen weltweit „versteckt und übersehen“ (S. 461) werden, Rebecca Yeo veröffentlichte 2015 einen Artikel unter der Überschrift „Disabled asylum seekers?... They don't really exist“ und Swantje Köbsell (2019) zeigt auf, dass behinderte Geflüchtete bislang weder in den Fachdiskursen noch in den Hilfesystemen in Deutschland wahrgenommen werden. Bezugnehmend auf diesen „blinden Fleck“ der Fluchtforschung und Disability Studies hat sich ein neues Forschungs- und Praxisfeld begonnen zu formen.

Der Anstieg der Zahlen von Schutzsuchenden in Deutschland in den Jahren 2015 bis 2016 (Bundesamt für Migration und Flüchtlinge, 2020) führte insbesondere auf der Ebene der Kommunen zur stärkeren Sichtbarkeit von praxisbezogenen Bedarfen von Geflüchteten ${ }^{1}$ mit Behinderung.

1 Aufgrund der Komplexität der Thematik verbunden mit der Notwenigkeit der Differenzierung agiert dieser Beitrag mit unterschiedlichen Begrifflichkeiten, die an dieser Stelle kurz geklärt werden sollen. Die Begriffe Geflüchtete und Schutzsuchende beziehen sich auf einen breiten Fluchtbegriff, sowohl Menschen im Asylprozess als auch abgelehnte und anerkannte Asylsuchende, sowie Personen die aufgrund anderer Schutzformen (z.B. humanitäre Aufnahmen und Resettlement) ein Bleiberecht erhalten haben werden in diesen beiden Begriffen zusammengefasst (siehe Statistisches Bundesamt, 2019, S. 5 sowie S. 251 dieser Publikation). Der Begriff 
Verbände, Organisationen der Selbsthilfe und die öffentliche Verwaltung griffen diese vermehrt auf und übersetzten sie in anwendungsbezogene Forschungsaktivitäten (AWO Bundesverband e.V., 2017; Leisering, 2018; Schwalgin \& Wank, 2017). Dieser Beitrag betrachtet die Situation in der Landeshauptstadt München. Bilder von neuankommenden Geflüchteten am Münchner Hauptbahnhof wurden 2015 weltweit medial verbreitet. Am ersten Septemberwochenende 2015 erreichten 17.500 Geflüchtete die Landeshauptstadt, ihre Unterbringung und Versorgung stand zunächst im Mittelpunkt der kommunalen Bemühungen (Korntheuer \& Hergenröther, 2020). Der vom ersten Bürgermeister 2016 beauftragte Gesamtplan zur Integration von Flüchtlingen (Landeshauptstadt München, Stelle für interkulturelle Arbeit, 2018, 2020) behandelte mit dem Ziel einer langfristigen Integrationsplanung auch Thematiken der (vulnerablen) Gruppen mit spezifischen Bedarfen. Erste Ansätze der Auseinandersetzung mit dem Feld Migration und Behinderung waren bereits innerhalb des Integrationskonzeptes von 2008 und in den städtischen Gremienstrukturen vorhanden. Es zeigte sich jedoch ein deutlicher Mangel an Erkenntnissen, welche die Größe der Bevölkerungsgruppe, die Unterbringungssituation und die Bedarfe von Geflüchteten mit Behinderung betreffen (Korntheuer, 2020)². Dieser Beitrag adressiert, basierend auf einer ersten explorativen Felderkundung und anwendungsbezogenen Abfragen, die folgenden leitenden Fragestellungen:

- Welche Aussagen können zur Anzahl der Menschen am Schnittpunkt von Flucht und Behinderung in München getroffen werden?

- Wie stellte sich die Wohn- und Lebenssituation von Geflüchteten mit Behinderung in Münchner Unterkünften zum Zeitpunkt der Befragungen (2016- 2018) dar?

- Welche ersten Hinweise können für eine inklusive Praxis formuliert werden?

In einem ersten Schritt werden die zentralen Begrifflichkeiten „Behinderung" und „Flucht" innerhalb der Fachdiskurse und der lokalen Zusammenhänge der Landeshauptstadt (LH) München verortet, um dann auf

Asylsuchende bezieht sich hingegen auf Personen im Asylverfahren. Neuzugewanderte sind Personen, die in den letzten vier Jahren nach Deutschland migriert sind, unabhängig von ihrem migrationsspezifischen und rechtlichen Status.

2 Eine erste Publikation zu den Ergebnissen der Abfragen wurde bereits 2020 in der Zeitschrift für Inklusion veröffentlicht: Korntheuer, Annette (2020): Intersektionale Ausschlüsse am Schnittpunkt Flucht und Behinderung. Erste Analysen in der Landeshauptstadt München. Zeitschrift für Inklusion (3). 
die komplexen Schnittpunkte und rechtlichen Zusammenhänge der beiden Felder einzugehen. Der derzeitige Forschungsstand wird im nächsten Schritt in Bezug auf zwei zentrale Zugänge fokussiert: einerseits theoretische Konzepte von Intersektionalität als Ausgangspunkt und andererseits anwendungsbezogene Forschungsaktivitäten. Zudem werden Forschungsergebnisse und -lücken bezüglich der Unterbringung von Geflüchteten mit Behinderung spezifiziert. Die methodische Umsetzung der Exploration des Feldes in der LH München folgt im vierten Absatz. Die aufgeführten Ergebnisse verdeutlichen die Zahl der Menschen am Schnittpunkt von Flucht und Behinderung und Ermöglichen eine Darstellung ihrer Wohnund Lebenssituation in München, was die Unterbringung, Aspekte des rechtlichen Status und Familienformen betrifft. Das Fazit bestätigt deutliche Bedarfe einer diversitäts- und intersektionalitätsbewussten Perspektive für Forschung und Praxis.

\section{Begriffliche und rechtliche Ausgangslagen}

\subsection{Kritisch reflektierte Adressierungen von Behinderung und Flucht}

Der von den Disability Studies und der UN-Behindertenrechtskonvention (UN BRK) angestoßene Diskurs zu Inklusion hat wichtige Impulse für die Weiterentwicklung des Verständnisses von Behinderung geliefert. Im herkömmlichen, medizinischen Modell von Behinderung werden körperliche oder seelische Beeinträchtigungen individualisiert und defizitorientiert betrachtet. Menschen sind behindert und müssen von Expert*innen innerhalb der vorhandenen Möglichkeiten gefördert werden. Paternalistische Grundannahmen von Fürsorge sind die Folge. Das soziale Modell von Behinderung setzt seinen Fokus hingegen auf die barrierereichen gesellschaftlichen Strukturen und Einstellungen, die die Teilhabe einschränken, also Menschen behindern (Hirschberg \& Köbsell, 2016, 556ff.). Theresia Degener (2016, S. 42) fügt schließlich auf Basis der UN BRK das Menschenrechtsmodell von Behinderung hinzu. Hier wird auch die gesellschaftliche Konstruiertheit von Behinderung betont. Binäre Vorstellungen müssen denen eines Kontinuums von Dis_ability ${ }^{3}$ weichen, um gesellschaftliche Marginalisierungs-, Stigmatisierungs- und Segregationsprozesse aufzubre-

3 Die Auflösung von binären Kategorien der Behinderung (Disability) und Befähigung (-ability) und die Annahme eines Kontinuums wird im Unterstrich von Dis_ability verdeutlicht. 
chen (Hirschberg \& Köbsell, 2016). Menschen mit Behinderung sind als Rechtsträger*innen und Expert*innen der eigenen Lebenssituation anzuerkennen. Weitreichende gesetzliche Umstrukturierungen durch das Bundesteilhabegesetz (BTHG) und die Novellierung des Behindertengleichstellungsgesetzes (BGG) sind in den letzten Jahren im Zuge des Nationalen Aktionsplans (NAP) der UN BRK angestoßen worden (Bundesministerium für Arbeit und Soziales, 2016, 25f.; Degener, 2016). In der LH München wurden verschiedene Aktionspläne verabschiedet, die inklusive Strukturen fordern und auf das soziale Modell von Behinderung zurückgreifen (Landeshauptstadt München, 2014; Landeshauptstadt München, Koordinierungsbüro zur Umsetzung der UN-Behindertenrechtskonvention, 2019).

Auch für den Bereich von Flucht zeigen sich Bezugspunkte zu den fluiden Grenzen von „Migrationskategorien“ auf wissenschaftlicher und kommunaler Ebene. "Flüchtlinge“ werden als rechtliche Kategorie auf der Grundlage der Genfer Flüchtlingskonvention definiert. Diese Definition berücksichtigt jedoch die Heterogenität und Vielfalt der Gründe und Prozesse, die Flucht verursachen, nur im eingeschränkten Maße. (Arbeits-)Migration und Zwangsmigration können nicht als binäre Gegensätze verstanden werden, da sich die Erfahrungen von existentieller Armut und bewaffneten Konflikten sowie die Hoffnung auf Zugang zu Bildung und wirtschaftlichem Wohlstand im Einzelfall oft überschneiden (Scherr \& Scherschel, 2019). Das Statistische Bundesamt in Deutschland verwendet den Begriff "Schutzsuchende", um eine breitere Kategorie zu definieren, die Asylsuchende, anerkannte Flüchtlinge, Menschen in Duldung sowie Resettlementflüchtlinge miteinschließt (Statistisches Bundesamt, 2019, S. 5). Auch der Münchner Gesamtplan zur Integration von Flüchtlingen agiert mit einem breiten Fluchtbegriff. Hier wurde ein komplexes Verfahren entwickelt, das über hundert verschiedene Rechtstitel berücksichtigt und diese zum Teil mit den Herkunftsländern kombiniert (Korntheuer \& Hergenröther, 2020; Landeshauptstadt München, Stelle für interkulturelle Arbeit, 2018).

\subsection{Komplex verwobene Rechtsbereiche im Feld von Flucht und Behinderung}

Am Schnittpunkt von Behinderung und Flucht treffen zwei Rechtsbereiche aufeinander, die häufig widersprüchlich oder zumindest nach unterschiedlichen Logiken funktionieren. Wie Matthias Otten et al. (2017) darstellen, kommt es zur „politische[n] Relativierung eines universalen Inklusionsparadigmas durch eine asylrechtliche Exklusionspraxis“ (S. 201). Der 
besondere Schutz der EU- Aufnahmerichtlinie (2013/33/EU) wird für Geflüchtete mit Behinderung häufig nicht umgesetzt und rechtliche Ansprüche auf Leistungen für Menschen mit Behinderung bleiben ihnen verwehrt (Westphal \& Wansing, 2019). Tatsächlich zeigen sich die rechtlichen Bedingungen am Schnittpunkt von Aufenthalts- und Asylrecht und den Leistungen der Rehabilitation und Teilhabe des Sozialgesetzbuches hochkomplex und ineinander verwoben. Die verpflichtende medizinische Erstuntersuchung für Asylsuchende nach $\$ 62$ Asylgesetz umfasst lediglich die Feststellung übertragbarer Krankheiten. Asylsuchende erhalten medizinische Behandlungen bei akuten Erkrankungen und Schmerzzuständen ( $\$ 4$ Asylbewerberleistungsgesetz (AsylbLG)), währenddessen stehen Eingliederungshilfen und Hilfen zur Pflege nach dieser Norm meist nicht zur Verfügung (Schülle, 2019, S. 150). Die Versorgung mit Hilfsmitteln, Hilfen zur Pflege und Rehabilitationsleistungen wird in den ersten 18 Monaten nach Ankunft durch die Sozialbehörden vor Ort sehr restriktiv geleistet, da sonstige Hilfen nur nach Ermessen der Sozialbehörden im Rahmen von Einzelfallentscheidungen bewilligt werden, wenn diese „zur Sicherung des Lebensunterhalts oder der Gesundheit unerlässlich“ ( $\$ 6$ Absatz 1 AsylbLG) sind. Für die Kommunen bestehen große Herausforderungen durch die mangelnde Qualifizierung der Sachbearbeiter*innen bezüglich des medizinischen und rehabilitationswissenschaftlichen Fachwissens (Leisering, 2018). Die rechtliche Ausgangslage und die Vielschichtigkeit der bürokratischen Ausgestaltung vor Ort zeigen sich als wesentliche Zugangsbarrieren. Diese Komplexität erhöht sich erneut, wenn neben Asylsuchenden andere Migrationskategorien, Aufenthaltsstatus und -dauern betrachtet werden (Gag \& Weiser, 2017; Schülle, 2019; Steiner, o.D.).

\section{Der Forschungsstand im Feld von Flucht und Behinderung}

\subsection{Intersektionalität als theoretisches Paradigma}

Die wissenschaftliche Auseinandersetzung mit dem Schnittpunkt von Flucht und Behinderung wird zum einen befördert durch den Aufstieg des Intersektionalitätsparadigmas in den Sozialwissenschaften, zum anderen ist die Aufmerksamkeit auch Folge einer stärkeren Ausdifferenzierung der Fluchtforschung (Kleist, 2019). In Anlehnung an Kimberlé Crenshaw (2013) analysiert das Konzept der Intersektionalität die Verwobenheit oder Überkreuzungen unterschiedlicher Differenzkonstruktionen (Walgenbach, 2016, S. 651; Winker \& Degele, 2009). Während in der Konstruktion sozialer Ungleichheitsverhältnisse die klassische Trias aus Ethnizität, Klasse 
und Geschlecht die zentralen Differenzlinien markiert, fand Behinderung als Differenzkategorie in der Intersektionalitätsforschung zunächst weniger Berücksichtigung (Wansing \& Westphal, 2014). Donja Armirpur (2016) verdeutlicht in ihren intersektionalen Fallstudien zu türkischen und iranischen Familien mit Kindern mit Behinderung die belastende Wirkung eines unsicheren rechtlichen Aufenthaltsstatus, den Ausschluss von Sozial- und Teilhabeleistungen und die schwierige Lebenssituation in Gemeinschaftsunterkünften für Geflüchtete (vgl. u.a. S. 142; S.179; S. 262). Flucht und Behinderung können in diverser Weise zusammenwirken. Behinderung kann sowohl ein Anlass als auch ein Hindernis für (Flucht)Migration sein. Seelische und körperliche Beeinträchtigungen können jedoch auch als Folge von Flucht entstehen (Pearce, 2015). Nach der Ankunft in (Erst-)Aufnahmeländern können Behinderungen ein Bleiberecht begründen, schützen aber laut Angela Grotheer und Joachim Schroeder (2019) in anderen Fällen nicht vor Abschiebung. Dennoch sind diese Zusammenhänge im Bereich der Fluchtforschung kaum adressiert worden. Es werden daher Forderungen geäußert, das theoretische Konzept von Intersektionalität „vom Kopf auf die Füße zu stellen“ und zu verdeutlichen, welche praktische Relevanz dieses Konzept für die Praxisfelder hat (Rathgeb, 2015, S. 88).

\subsection{Anwendungsbezogene Bearbeitung des Feldes als „praktische Notwendigkeit"}

In Folge des „langen Sommers der Migration“ (Hess et al., 2017) war eine starke Expansion von Angeboten der Migrations- und Integrationsarbeit zu beobachten (Korntheuer \& Hergenröther, 2020). Eine aktuell deutlich rückläufige Zahl von neu registrierten Schutzsuchenden trifft daher nun auf ein breit aufgestelltes Praxisfeld. Neben der verstärkten Sichtbarkeit aufgrund der Fluchtzuwanderung in den Jahren 2015 und 2016 können Spezialisierungen und zahlreiche Praxis- und Vernetzungsinitiativen ${ }^{4}$ in

$4 \mathrm{Zu}$ nennen sind hier unter anderem das Projekt "ZurFlucht Lebenshilfe“ in Hamburg, Intitiativen der Gehörlosenverbände, die Beratungsstelle „ComIn“ von „Handicap International“ in München, die Fachstelle für Flüchtlinge mit Behinderung und ältere Flüchtlinge im Berliner Netzwerk, die Fachtagungen und Workshops des Vereins „Mina Leben in Vielfalt“ in Berlin sowie das bundesweit aktive Projekt „Crossroads. Flucht, Migration, Behinderung“ von „Handicap International“. Quellen: Korntheuer (2020); Grotheer (August/2017); Handicap Interantional (2019); Otten, Schahrzad und Zuhr (2017). 
diesem Zusammenhang auch Reaktionen der Praxis- und Trägerlandschaft auf eine nun gesunkene Nachfrage an ihren Integrationsangeboten sein, um neue Teilnehmer*innen zu gewinnen.

Beispiele für eine praxisbezogene Forschung sind die Abfragen des Caritas Verbandes (Steiner, o.D.) und der Arbeiterwohlfahrt (AWO Bundesverband e.V., 2017) sowie eine explorative Studie zu den Lebensbedingungen von Geflüchteten mit Behinderung in Hamburg (Grotheer, 2017; Grotheer \& Schroeder, 2019). Auch die Bedarfsanalyse des Deutschen Institutes für Menschenrechte (Schwalgin \& Wank, 2017) und der Teilhabebericht der Bundesregierung (Bundesministerium für Arbeit und Soziales, 2016) zeigen erste Erkenntnisse bezüglich der Schnittstelle Flucht und Behinderung. Die Monitoringstelle zur UN-BRK (Leisering, 2018) und die Fachverbände für Menschen mit Behinderung (2019) haben zudem Positionspapiere mit konkreten Handlungsempfehlungen veröffentlicht.

Die Versäulung der Angebotslandschaft in Behindertenhilfe, Migrations- und Integrationsarbeit, intransparente und restriktive rechtliche und bürokratische Prozesse, Informationsdefizite sowie die mangelnde Barrierefreiheit von Unterbringung und Angeboten (z.B. fehlende Sprachmittlung) werden als zentrale Hindernisse für die gesellschaftliche Teilhabe von Geflüchteten mit Behinderung benannt (AWO Bundesverband e.V., 2017; Steiner, o.D.). Wie die Abfrage von Beratungsdiensten der Caritas zeigt, werden diese Hindernisse insbesondere im Feld der Behindertenhilfe deutlich. 72\% der Berater*innen in der Behindertenhilfe geben fehlendes Wissen bezüglich des Leistungsanspruches als Hindernis an (Steiner, o.D.). Die interne Abfrage von Fachkräften und Ehrenamtlichen der Arbeiterwohlfahrt zeigt mit 484 angegebenen Adressat*innen am Schnittpunkt von Flucht und Behinderung in 24 Institutionen eine hohe Bedeutung der Thematik und betont die fehlende Identifizierung und Diagnostik bereits als wesentliches Exklusionsmoment. Das Ehrenamt hingegen bemüht sich um Abfederung der fehlenden öffentlichen Inklusionsstrukturen und um Sicherstellung eines Minimums an Teilhabe (AWO Bundesverband e.V., 2017)

\subsection{Wobn- und Lebenslagen in Unterkünften}

Anwendungsbezogene Studien, Abfragen und Positionspapiere am Schnittpunkt Flucht und Behinderung nehmen die barrierefreie Unterbringung in Unterkünften in den Blick, können im Augenblick jedoch nur selten auf empirische Ergebnisse und klare Datenlagen zurückgreifen (AWO Bundesverband e.V., 2017; Die Fachverbände für Menschen mit 
Behinderung, 2019; Grotheer, August/2017; Grotheer \& Schroeder, 2019; Leisering, 2018; Schwalgin \& Wank, 2017; Steiner, o.D.).

Es kommt in den Ländern und Kommunen zu keiner systematischen Erfassung von Beeinträchtigungen bei neuzugewiesenen Geflüchteten. Auch vereinzelte barrierefreie Plätze in Sammelunterkünften sind meist nicht zentral registriert. Zudem wird Barrierefreiheit häufig auf die $\mathrm{Zu}$ gänglichkeit für Menschen mit Mobilitätsbeeinträchtigungen reduziert, während notwendige Rahmenbedingungen für Menschen mit Sinnesbeeinträchtigungen oder kognitiven und psychischen Beeinträchtigungen ohne Beachtung bleiben (Leisering, 2018). Kenan Engin (2019) führt Mängel der barrierefreien Unterbringung und unzureichende Unterstützungsleistungen insofern in erster Linie auf mangelnde Identifizierung und Informationsweitergabe zurück. Der Asylsozialarbeit in den Unterkünften kommt in der Identifikation von Bedarfen und bezüglich des Zuganges zu Ressourcen eine Schlüsselrolle zu. Begrenzt wird dies allerdings durch die Aussage der Arbeiterwohlfahrt:

„Weiterhin ist auch die Wohnsituation in Erstaufnahmeeinrichtungen und Gemeinschaftsunterkünften oft nicht geeignet eine Umgebung zu bieten, die ein Clearing begünstigt. Der Betreuungsschlüssel von Sozialarbeiter*innen in zentralen, sowie auch dezentralen Unterkünften ist meist zu hoch, so dass die nötigen Kapazitäten nicht vorhanden sind, um mit allen Bewohner*innen ausführliche Gespräche zu führen.“ (AWO Bundesverband e.V., 2017, S. 14)

Ein Positionspapier der Behindertenverbände sieht dringenden Handlungsbedarf, da insbesondere in den von der Bundesregierung verabschiedeten und seit 2018 eingerichteten Zentren für Ankunft, Entscheidung, Rückführung (AnKER-Zentren) notwendige Strukturen und Ressourcen zur Identifikation und bedarfsgerechten Unterstützung nicht hinreichend gegeben sind (Die Fachverbände für Menschen mit Behinderung, 2019).

Die „Mindeststandards zum Schutz von geflüchteten Menschen in Flüchtlingsunterkünften“ (BFSFJ \& UNICEF 2018) gehen innerhalb eines eigenen Anhangs auf die spezifischen Schutzbedarfe von Geflüchteten mit Behinderung ein. Sie sehen Bedarfe vor allem im Zugang zu Identifizierung und Diagnostik und weisen auf die Notwendigkeit der Fortbildung und Sensibilisierung beim Personal hin. Menschen mit Behinderung attestieren sie ein besonderes Risiko für Diskriminierungs- und Gewalterfahrungen. Eine umfassende barrierefreie Unterbringung und die stärkere Einbindung von externen Kooperationspartner*innen (z.B. der Behindertenhilfe und Selbsthilfe) werden als weitere Standards eingefordert (ebd., S. 38ff). 
Auch eine explorative Studie an der Universität Hamburg kann nur auf vereinzelte und fragmentierte Datenquellen verweisen, zeigt jedoch wesentliche Erkenntnisse zu den kommunalen Strukturen und Diskursen auf (Grotheer 2017, S. 30). Die kommunalen Verwaltungsstrukturen, Akteursebenen und Prozesse sind demnach äußerst komplex, dennoch versuchen Akteur*innen die Thematik in die Abläufe und im Bereich der Unterbringung zu integrieren:

„(...) man ist bemüht, zumindest bei der Zuweisung in Erstaufnahmeund Folgeunterkünften (dazu zählen auch Abteilungen in Krankenhäusern und Pflegeheimen), die individuellen Lebenssituationen und spezifischen Bedürfnisse beeinträchtigter Geflüchteter so gut es geht zu berücksichtigen“" (Grotheer 2017, S. 40).

Von einem ähnlichen Vorgehen einer Erstaufnahmeunterkunft in Thüringen berichtet auch Engin (2019). Für Grotheer steht einer durchgängige Orientierung an den Bedarfen und Rechten der Personen mit Behinderungen die nicht stattfindende, systematische Erfassung der Personen und das mangelnde Fachwissen der Mitarbeiter"innen in den Unterkünften entgegen. Auf Ebene der Bezirke, die wichtige Aufgaben des Sozialraummanagements übernehmen, zeigt sich zudem wenig Bewusstsein bezüglich des Themas Flucht und Behinderung, auch wenn Mitarbeiter*innen an der Basis berichten, im Arbeitsalltag mit unterschiedlichsten Formen von Behinderung Erfahrungen zu machen (Grotheer 2017, S. 43). Aus Praxissicht sind Strukturen und Zuständigkeiten wenig transparent (ebd. S. 55). So kann zusammengefasst werden, dass für deutsche Kommunen bis dato keine Zahlen zu Geflüchteten mit Behinderung in Unterkünften vorlagen und sich auch sonst eine empirisch fundierte Erkenntnislage zu ihren Wohn- und Lebenslagen als äußerst mangelhaft darstellt.

\section{Methodische Umsetzung der Exploration des Feldes}

\subsection{Erkenntnisleitende Fragestellungen und Datenerhebung}

Als Bildungskoordinatorin für Neuzugewanderte mit besonderen Bedarfen $^{5}$ war es mir möglich, innerhalb der Landeshauptstadt München eine

5 Da die Tätigkeit als Bildungskoordinatorin an der Analyse von Neuzugwanderten mit spezifischen Bedarfen ausgerichtet war, bezogen sich die Erhebungen hier auf Neuzugewanderte mit Beeinträchtigungen und chronischen Erkrankungen sowie 
erste Exploration des Feldes unter einer stark anwendungsbezogenen Perspektive im Zeitraum von 2016 bis 2018 vorzunehmen. Die Ergebnisse sollten direkt für die Konzeption von bedarfsgerechten Angeboten innerhalb der Stadtverwaltung verwendet werden und fanden unter anderem Eingang in das Projekt „Münchner Gesamtplan zur Integration von Flüchtlingen“ (Landeshauptstadt München, Stelle für interkulturelle Arbeit, 2018). Erkenntnisleitende Fragestellungen der Erhebungen basierten auf dieser anwendungsbezogene Zielformulierung. Die vorhandene Angebotslandschaft in München und die von Expert"innen und Neugzugewanderten genannten Bedarfe sollten exploriert und anhand der Analyse vorhandener Ressourcen und (Zugangs)-Barrieren Ideen, Handlungsempfehlungen und Konzeptionen entworfen werden. Fokus der Erhebungen waren Bildungs- und Integrationsangebote; die Wohn- und Lebenssituation in den Unterkünften zeigte sich jedoch als ein zentraler Einflussfaktor auf Teilhabeprozesse und wurde deshalb mit in den Blick genommen.

Die Datenerhebung fand in mehreren Schritten statt:

- Zunächst erfolgte eine explorative Erkundung des Feldes in der Landeshauptstadt durch 30 qualitative Interviews und informelle Gespräche mit Expert"innen im Feld der Integrationsarbeit ${ }^{6}$ (Kruse, 2015), um eine systematische Erfassung der Bildungsbedarfe und Bildungsangebote von neuzugewanderten Menschen mit Behinderungen vorzubereiten. Zentrale Ergebnisse wurden festgehalten und innerhalb eines Workshops mit 45 Teilnehmenden (Expert*innen und Neuzugewanderten) ergänzt. Expert"innen waren unter anderem Integrationskursträger oder im Bereich der Migrationsberatung, Asylsozialarbeit, Teilhabeberatung und im mobilen sonderpädagogischen Dienst tätig.

- Die mangelnde Barrierefreiheit von Bildungsangeboten zeigte sich innerhalb der ersten explorativen Phase als wesentliche Barriere für den Zugang zu Bildung und wurde deshalb im zweiten Schritt durch eine Abfrage mithilfe von Fragebögen der Sprach- und Integrationskursträger genauer erhoben. Hierzu liegen Rückmeldungen von 34 Standorten aus dem Jahr 2017 vor.

- Da in der Stadtverwaltung keine Informationen zur Anzahl der Menschen am Schnittpunkt von Flucht und Behinderung vorlagen, wurden in einem dritten Schritt Bedarfe durch eine Abfrage der Asylsozialdienste in allen 41 Münchner Unterkünften für Geflüchtete von April

auf neuzugewanderte Frauen mit Kinderbetreuungsbedarfen und neuzugewanderte LGBTIQ*.

6 Siehe Fußnote 5, S. 157f. 
bis August 2018 ermittelt und auch Angaben zu ihrer Lebens- und Wohnsituation, sowie ihren Bildungsbedarfen abgefragt. Da davon auszugehen war, dass dennoch nur eine Teilgruppe der Geflüchteten mit Behinderung durch die Abfrage der Unterkünfte zu erreicht werden konnte, analysierte ich zudem die Daten des Zentrums Bayerns Familie und Soziales (ZBFS) zu Drittstaatsangehörigen mit Schwerbehindertenausweis.

Für die Abfrage der Unterkünfte im dritten Schritt der Erhebung (Erstaufnahme, staatliche und sogenannte dezentrale Unterkünfte in kommunaler Trägerschaft ${ }^{7}$ ) wurden Mitarbeiter*innen der Asylsozialdienste gebeten, Personen innerhalb vier Kriterien anzugeben: i)Personen mit diagnostizierter Behinderung/Erkrankung; ii) Personen, die aufgrund der angegebenen Beeinträchtigung in ärztlicher/therapeutischer Behandlung waren; iii) Personen, die aufgrund der angegebenen Beeinträchtigung mit dem Asylsozialdienst mehrfach in Kontakt waren und an entsprechende Dienste/Ärzt*innen verwiesen wurden; vi) Personen mit offensichtlichen Beeinträchtigungen. Zudem wurde in einem zweiten Schritt mittels einer telefonischen Nachfrage geprüft, ob die Angaben den Kriterien gemäß erfolgt waren, oder ob Unklarheiten bei den Asylsozialdiensten bestanden hatten. Da die Abfrage über die kommunale Fachsteuerung zugeleitet wurde, war es möglich, von allen Unterkünften Antworten zu erhalten. Die Zahlen stellen dementsprechend eine repräsentative Vollerhebung der Münchner Unterkünfte zu diesem Zeitpunkt dar. Personen mit Beeinträchtigungen, die nicht in der Beratung der Asylsozialdienste waren, wurden jedoch nicht erfasst.

\subsection{Datenauswertung und Methodenkritik}

Die Auswertung der quantitativen Daten der Abfrage von Unterkünften für Geflüchtete wurde unter der Zielsetzung von anwendungsbezogener deskriptiver Statistik mit der Software SPSS vorgenommen und durch die Auswertung der Zahlen des ZBFS ergänzt. Als weitere Quellen wurden die qualitativen Daten thematisch kodiert und durch Berichterstattungen der LH München zum Gesamtplan zur Integration von Flüchtlingen komplettiert (Stelle für interkulturelle Arbeit, 2018; 2020).

7 Auch bei der dezentralen kommunalen Unterbringung handelt es sich zum Großteil um Gemeinschaftsunterkünfte mit ca. 50 bis zu 350 Bettplätzen. 
Forschung an intersektionalen Schnittstellen birgt Risiken für Forschende und Forschungsteilnehmende (Korntheuer et al., 2021; Otten, 2019). Praxisakteur*innen fordern zwar zur Erhöhung der Handlungsfähigkeit eine konkrete Datenbasis und Aussagen zur Anzahl der Menschen am Schnittpunkt von Flucht und Behinderung in Deutschland (Die Fachverbände für Menschen mit Behinderung, 2019). An den hier vorliegenden quantitativen Abfragen und Analysen kann jedoch kritisiert werden, dass nur strukturelle Dimensionen in den Blick genommen wurden und Diskurse sowie subjektive Deutungen der Geflüchteten mit Behinderungen nicht zum Tragen kommen. Es besteht die Gefahr einer unkritischen Reproduktion und somit einer Essentialisierung von „Flüchtlingen“ und „Behinderten“, wenn Kategorien in statistischen Erhebungen nicht hinterfragt werden und gesellschaftliche Diskurse ohne Berücksichtigung bleiben (siehe 2; Korntheuer, 2020; Korntheuer et al., 2021). Goodley (2015) spricht daher von den „Rändern der Disziplinen“ (S. 44), an denen sich Forscher*innen bewegen, die sich mit den verwobenen Prozessen von Migration und Behinderung auseinandersetzen. Die Diversität der Lebenssituationen von Geflüchteten mit Behinderung kann durch die Erhebung der subjektiven Deutungen, von Betroffenen selbst stärker in den Fokus gerückt werden. Partizipative Forschungsmethoden hätten hier neue Möglichkeitsräume eröffnen können ${ }^{8}$.

\section{Darstellung und Diskussion der Ergebnisse}

\subsection{Zablen zu Geflüchteten mit Behinderung in Münchner Gemeinschaftsunterkünften}

Insgesamt wurden in der Abfrage 801 Personen mit Beeinträchtigungen in Münchner Unterkünften angegeben. Bei 7631 Bewohner*innen, die zum Stand Dezember 2017 in den Münchner Unterkünften lebten, entsprechen 801 genannte Personen mit Beeinträchtigungen und Erkrankungen 10\% der gesamten Bewohner*innen (Korntheuer, 2020). Mit Blick auf die Arten der Beeinträchtigungen zeigt sich in der Abfrage der Unterkünfte, dass mit 127 Personen eine hohe Zahl von Menschen mit Mehrfachbeeinträchtigungen untergebracht war. Insgesamt wurden 982 Beeinträchtigungen bei 801

$8 \mathrm{Zu}$ den Möglichkeiten und Grenzen von partizipativen Forschungsmethoden am Schnittpunkt von Migration und Behinderung siehe ausführlich Korntheuer, Afeworki Abay und Westphal (2021). 
Personen angegeben (siehe Tabelle 1). Psychische Erkrankungen wurden für die Altersgruppe der 18 bis 49-jährigen als häufigste Art der Beeinträchtigung genannt. Eine Differenzierung nach Arten von psychischen Erkrankungen war in einem Anmerkungsfeld möglich, benannte Erkrankungen waren hier neben Traumafolgestörungen auch Depressionen und Suchterkrankungen. Alle Altersgruppen waren zudem stark von chronischen Erkrankungen betroffen. Für Personen über 64 Jahren wurden prozentual häufiger Beeinträchtigungen des Bewegungsapparates angegeben. Bei Kindern und Jugendlichen war der Wert bezüglich Lern- und geistiger Behinderung höher als bei Erwachsenen. Es ist anzunehmen, dass dies an der höheren Diagnosewahrscheinlichkeit im schulischen Kontext lag.

\section{Tabelle Nr. 1 Arten der angegebenen Beeinträchtigungen nach Alter}

\begin{tabular}{|c|c|c|c|c|c|c|c|}
\hline Alter & $>18$ & $18-24$ & $25-49$ & $50-64$ & $<64$ & k.A. & Gesamt \\
\hline $\begin{array}{l}\text { psychische } \\
\text { Erkrankung }\end{array}$ & 31 & 99 & 157 & 10 & 4 & 2 & 303 \\
\hline $\begin{array}{l}\text { psychische } \\
\text { Behinderung*** }\end{array}$ & 12 & 26 & 46 & 4 & & & 88 \\
\hline $\begin{array}{l}\text { chronische } \\
\text { Erkrankung }\end{array}$ & 51 & 57 & 133 & 29 & 20 & 1 & 291 \\
\hline $\begin{array}{l}\text { Beeinträchtigung des Be- } \\
\text { wegungsapparates }\end{array}$ & 28 & 9 & 42 & 15 & 14 & & 108 \\
\hline Hörbeeinträchtigung & 5 & 2 & 3 & & 1 & & 11 \\
\hline Sehbeeinträchtigung & 15 & 3 & 17 & 7 & 2 & & 44 \\
\hline $\begin{array}{l}\text { geistige } \\
\text { Behinderung* }\end{array}$ & 24 & 5 & 2 & 2 & 1 & & 34 \\
\hline Lernbehinderung* & 60 & 14 & 11 & 3 & 1 & & 89 \\
\hline k.A./ andere & 1 & 3 & 8 & 1 & & 1 & 14 \\
\hline Gesamt & 227 & 218 & 419 & 71 & 43 & 4 & 982 \\
\hline
\end{tabular}

Quelle: Eigene Darstellung, Abfrage des Sozialreferats LH München 2018; **=die Anzahl wurde um 44 Doppelangaben von psychischer Behinderung und psychischer Erkrankung bereinigt; psychische Behinderung wurde als psychische Erkrankung definiert, die länger als 6 Monate andauert " $=$ es wurden bei 20 Personen eine Doppelangabe von Lernbehinderung und geistiger Behinderung vorgenommen. 
Zur Quantifizierung der Bedarfe am Schnittpunkt von Flucht und Behinderung in der Kommune München wurden zudem die Zahlen des Zentrum Bayerns für Familie und Soziales (ZBFS) zu Inhaber*innen von Schwerbehindertenausweisen ausgewertet. Diese Daten verdeutlichen, dass neben den aktuellen Bewohner*innen der Gemeinschaftsunterkünfte von weiteren Personen auszugehen ist, die entweder im Bereich der Wohnungslosenhilfe in Unterkünften leben oder aber bereits in privaten Wohnraum umgezogen sind. Auch diese Personen, so ist anzunehmen, sind oder waren zum Großteil von der Unterbringung in Gemeinschaftsunterkünften betroffen, wenn sie als Schutzsuchende nach Deutschland gekommen sind.

Die vorliegenden Daten des ZBFS ermöglichen eine Differenzierung nach Staatsangehörigkeit, Geschlecht und befristetem bzw. unbefristetem Aufenthaltsstatus. Ende 2019 lebten in der Landeshauptstadt München 25.825 Personen mit Schwerbehindertenausweis und ausländischer Staatsbürgerschaft. Im Vergleich der beiden Jahre 2017 und 2019 zeigt sich ein deutlicher Anstieg der Gesamtgruppe von 8,6\% und eine leichte Zunahme des Anteils von Personen mit befristetem Aufenthaltsstatus. Der Anteil von weiblichen und männlichen Personen betrug 2019 jeweils 50\%.

Abbildung Nr. 1. Personen mit ausländischer Staatsbürgerschaft und Schwerbehindertenausweis, LH München, 2017; Quelle: ZBFS, 2020; "enthält auch Zablen zu EU Personen mit unklarer Aufenthaltsberechtigung

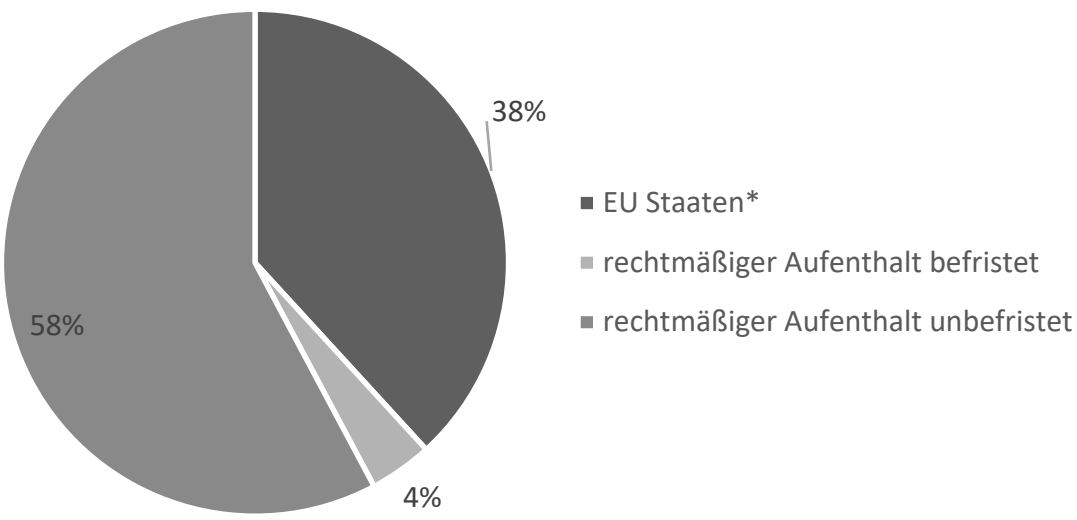


Abbildung Nr. 2. Personen mit ausländischer Staatsbürgerschaft und Schwerbehindertenausweis, LH München, 2019; Quelle: ZBFS, 2020; "enthält auch Zablen zu Personen mit unklarer Aufenthaltsberechtigung

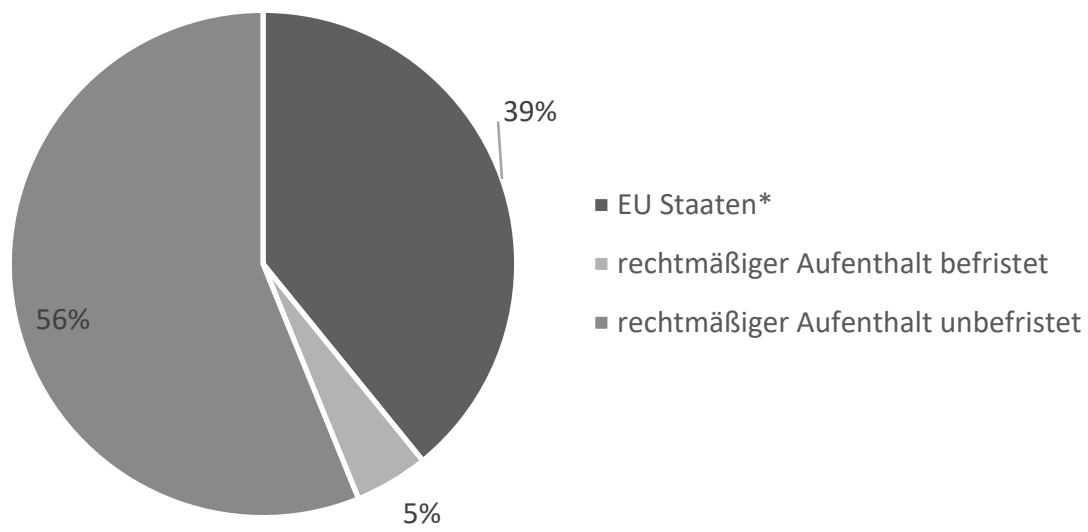

Afghanistan, Irak, Syrien, Nigeria und Somalia sind die fünf meist vertretenen Herkunftsländer von Geflüchteten in München. Ende 2018 lebten über 11.000 Personen mit Fluchterfahrung in München, die zwischen 2014 und 2018 aus diesen fünf Ländern eingereist waren. Sie stellen 74,8\% der in diesen Jahren zugewanderten Fluchtbevölkerung dar (Interkulturelle Stelle, 2020, S. 29). Ein deutlicher Anstieg von Personen mit Schwerbehindertenausweis aus diesen Fluchtherkunftsländern wird in der Auswertung der Zahlen des ZBFS sichtbar (siehe Abbildung 3), dennoch ist anzunehmen, dass ein Teil dieser Menschen bereits vor 2014 nach München eingereist war. Hinweise auf den erschwerten Zugang zum Schwerbehindertenausweis zeigen sich in der Abfrage der Unterkünfte. 801 Personen mit Beeinträchtigungen wurden hier angegeben. Lediglich 104 dieser Personen hatten einen Schwerbehindertenausweis erhalten, beantragt waren weitere 26 . 
Abbildung Nr. 3. Personen mit Schwerbehindertenausweis aus fünf Hauptfluchtherkunftsländern, LH München, 2017-2019; Quelle: ZBFS, 2020, jeweils zum 31.12. des Jahres; * Gesamt zu den fünf Hauptfluchtherkunftsländern; Daten zu weiteren Herkunftsländern von Geflüchteten sowie genauer Aufenthaltsstatus liegen im Datensatz des ZBFS nicht vor.

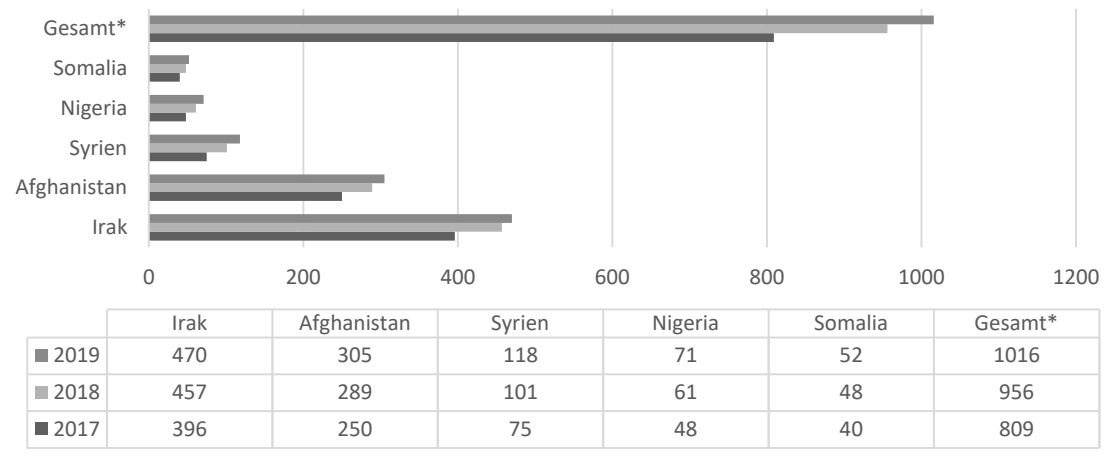

5.2 Die Wohn- und Lebenssituation von Geflüchteten mit Behinderung in Münchner Unterkünften

Geflüchtete mit Behinderung in unterschiedlichen Unterbringungsformen Geflüchtete und Asylsuchende leben in München in unterschiedlichen Wohn- und Unterbringungsformen: in Erstaufnahmeeinrichtungen (sowie ab 2018 in Dependancen des AnKER Zentrums Manching), Gemeinschaftsunterkünften sowie in der dezentralen kommunalen Unterbringung (siehe Fußnote 7, S. 159). Ende 2018 waren 7.106 Menschen in diesen Wohnformen untergebracht, hiervon waren etwa 2.684 anerkannte Flüchtlinge und subsidiär Schutzberechtigte. Geflüchtete haben nach dem Erhalt einer Aufenthaltserlaubnis die rechtliche Möglichkeit, aus den Unterkünften auszuziehen, aufgrund der angespannten Situation auf dem Münchner Wohnungsmarkt stellt der Zugang zu Wohnraum jedoch eine enorme Herausforderung dar. Auch im Bereich der akuten Wohnungslosenhilfe nimmt der Anteil von Personen aus den Hauptherkunftsländern von Geflüchteten ständig zu und betrug im Dezember 2018 bereits 39\% (Landeshauptstadt München, Stelle für interkulturelle Arbeit, 2020, S. 35). Leider konnten in der hier geschilderten Abfrage jedoch keine Daten zu Geflüchteten mit Behinderung im Bereich der Wohnungslosenhilfe erhoben werden. 
Um den Anforderungen der EU-Aufnahmerichtlinie für die Unterbringung von besonders schutzbedürftigen Geflüchteten (2013/33/EU) zu entsprechen, erstellte die Stadtverwaltung 2019 ein ganzheitliches Gewaltschutzkonzept. Dieses findet jedoch keine Anwendung in landeseigenen Gemeinschaftsunterkünften und den 2018 eingeführten AnKER-Einrichtungen, da die Kommune hier nicht zuständig ist (Landeshauptstadt München, Stelle für interkulturelle Arbeit, 2020, S. 40). Im Stadtgebiet von München existieren zwei Dependancen des AnKER-Zentrums Manching, die Funkkaserne und die Unterkunft am Moosfeld. Neu ankommende Asylsuchende fallen in dieser Unterbringungsform in die Zuständigkeiten der jeweiligen Regierungsbezirke und sollen dort bis zum Ende ihres Asylverfahrens (höchsten 24 Monate) leben. Aus dem AnKER Zentrum in der Funkkaserne sind Berichte der LH München von Ende 2018 vorhanden. Die sogenannte verdichtete Unterbringung von 281 Personen (hiervon 188 Kinder) wirkte sich in Form einer zunehmenden Kindeswohlgefährdung und massiven psychischen Belastungen aus. Der Zugang von externen Akteur*innen zur Unterkunft, wie Beratungsstellen, war zudem stark eingeschränkt (Landeshauptstadt München, Stelle für interkulturelle Arbeit, 2020, S. 19). Die von den Fachverbänden geäußerten Befürchtungen und die Kritik an dieser Unterbringungsform bezüglich fehlender Identifikation, Diagnostik und Unterstützung von Geflüchteten mit Behinderung durch Hilfenetzwerke zeigen sich in München bereits bestätigt (siehe 2.3). Da die Abfrage in den Münchner Unterkünften zwischen April und August 2018 durchgeführt wurde, waren Erstaufnahmeunterkünfte zu diesem Zeitpunkt noch nicht in AnKER-Dependancen transformiert worden.

\section{Heterogene Problemlagen bei der Unterbringung in Gemeinschaftsunterkünften}

Von den 801 benannten Personen mit Beeinträchtigungen in den Münchener Unterkünften wurde nur bei 247 Personen die Art der Unterbringung als unproblematisch eingeschätzt. Gerade für Personen mit chronischen Erkrankungen und psychischen Beeinträchtigungen stellten Mehrbettzimmer laut der Befragung eine hohe Belastung dar. Zudem wurde in diesem Zusammenhang angegeben, dass für 100 Personen kein $\mathrm{Zu}$ gang zu psychiatrischer Versorgung bestand. Unzureichende barrierefreie Unterbringung und schlechte Verkehrsanbindung sind vor allem für Personen mit Mobilitätseinschränkungen und Sinnesbeeinträchtigungen relevant. Obwohl es insgesamt 163 Angaben von Beeinträchtigungen des Bewegungsapparates, des Sehens und Hörens gab, wurde die mangelnde Barrierefreiheit nur in 51 Fällen als problematisch angesehen. Eine Erklärung hierfür kann eine Reduzierung des Verständnisses von Barrierefreiheit bei den befragten Asylsozialdiensten auf die Bedarfe von Rollstuhlnutzer*in- 
nen sein. Der Begriff der Barrierefreiheit spielt eine zentrale Rolle bei der Umsetzung von inklusiven Strukturen im Sinne der UN-BRK (siehe 2), er wird im Behinderungsgleichstellungsgesetz (BGG) folgendermaßen definiert:

„Barrierefrei sind bauliche und sonstige Anlagen, Verkehrsmittel, technische Gebrauchsgegenstände, Systeme der Informationsverarbeitung, akustische und visuelle Informationsquellen und Kommunikationseinrichtungen sowie andere gestaltete Lebensbereiche, wenn sie für Menschen mit Behinderungen in der allgemein üblichen Weise, ohne besondere Erschwernis und grundsätzlich ohne fremde Hilfe auffindbar, zugänglich und nutzbar sind.“( $\$ 4$ BGG)

Geschaffene Angebote wie rollstuhlgerechte Appartements wurden nur sehr zögerlich oder gar nicht in Anspruch genommen, obwohl mobilitätsbeeinträchtigte Personen in barrierereichen Unterkünften lebten (Landeshauptstadt München, Stelle für interkulturelle Arbeit, 2020, S. 39). Die Abfrage verdeutlicht hier, dass, wie bereits im Forschungsstand (siehe 3) beschrieben, auch in München keine wirksamen Prozesse der Identifikation und Informationsweitergabe sowie keine transparenten Zugangswege vorhanden waren.

Abbildung Nr. 4 Problematische Aspekte der Unterbringung in Unterkünften Quelle: Eigene Darstellung, Abfrage des Sozialreferats LH München 2018

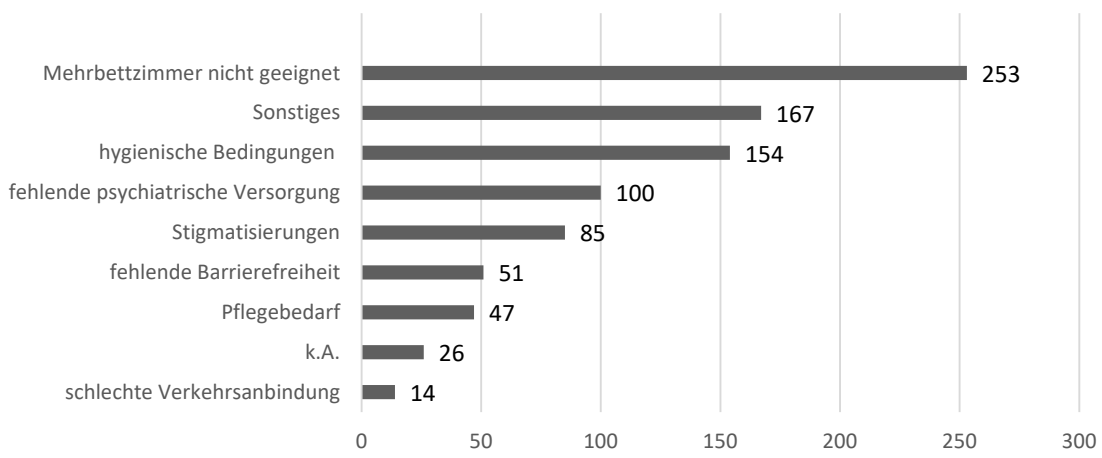

Ausschlussprozesse aufgrund des rechtlichen Status

Der rechtliche Status bestimmt die Lebenssituation und gesellschaftlichen Teilhabechancen von Geflüchteten. Der Schnittpunkt von Asyl- und Aufenthaltsrecht sowie Rehabilitationsrecht zeigt sich außerordentlich 
komplex; neben dem rechtlichen Status spielt auch die Aufenthaltsdauer eine wichtige Rolle (siehe 2). Ein Großteil der durch die Asylsozialarbeit benannten Personen $(87,5 \%)$ lebte bereits länger als 15 Monate in München. Der Wartezeitraum für eine Gleichstellung mit gesetzlich krankenversicherten Personen wurde im August 2019 um 3 Monate auf nun 18 Monate verlängert ( $\mathbb{2}$ Abs. 1 AsylbLG). Zum Zeitpunkt der Abfrage bestand somit zumindest rechtlich in Bezug auf die Gesundheitsversorgung ein gleichgestellter Zugang für diese 702 Bewohner*innen. Das Referat für Gesundheit und Umwelt (RGU) der LH München bietet zudem seit 2016 einen aufsuchenden medizinischen Dienst in Unterkünften für Geflüchtete an, um den Zugang zu Gesundheitsleistungen zu unterstützen (Landeshauptstadt München, Referat für Gesundheit und Umwelt).

Der Teilhabebericht der Bundesregierung nimmt eine strukturelle Unterversorgung im Rahmen des Asylbewerberleistungsgesetzes an. Jedoch nicht nur rechtliche Ausschlüsse bilden Zugangshindernisse auch Informationsdefizite, Kommunikationsprobleme und mangelnde Kultursensibilität führen zur geringeren Teilhabe an Rehabilitationsleistungen (Bundesministerium für Arbeit und Soziales, 2016). 325 Personen (40,5\%) in der Münchner Abfrage besaßen bereits eine Aufenthaltserlaubnis, während 97 Personen (12\%) eine Duldung erhalten hatten und sich 329 (41\%) noch im Asylverfahren befanden. Im Gesamtblick zeigen die Interviews und Expert"innengespräche hohe bürokratische und sprachbedingte Hürden im Hinblick auf die Gesundheitsversorgung und den Zugang zu Rehabilitationsleistungen von Geflüchteten mit und ohne Aufenthaltserlaubnis. Diese spiegeln sich auch in der Abfrage der Unterkünfte wider. 292 Personen $(36,4 \%)$ hatten neben der Beratung durch die Asylsozialarbeit zum Zeitpunkt der Abfrage keinen Zugang zu weiteren Unterstützungsmaßnahmen und Bildungsangeboten. Abgefragt wurden hier die Teilnahme an Sprach- und Integrationskursen sowie weiteren Bildungsangeboten, die Beratung durch Handicap International und sonstige Maßnahmen und Beratungen. Mangelnde Barrierefreiheit und unzureichende Kennzeichnungen von Angeboten zeigen sich in der Abfrage der Integrations- und Sprachkurse als institutionelle Hürden der Teilhabe.

\section{Differenzierung nach Alter und Familiensituation}

Kinder mit Beeinträchtigungen erhielten im Vergleich zu Erwachsenen häufiger Unterstützungs- und Bildungsangebote, was im Zusammenhang mit der gesetzlichen Schulpflicht stehen kann. Dennoch nahmen 20 Kinder und Jugendliche mit Beeinträchtigungen im Alter bis 16 Jahre neben der Asylsozialberatung an keinen weiteren Maßnahmen und Unterstützungen teil. Die Expert*innen betonten, dass, obwohl zum Beispiel Früh- 
förderung über den $\$ 6$ AsylBLG möglich wäre, Informationsbarrieren und aufwendige bürokratische Abläufe einen schnellen Zugang erschweren. Auch der Zugang zu Sprachkursen und Berufsintegrationsklassen bei Jugendlichen und jungen Erwachsenen mit Beeinträchtigungen war eingeschränkt. 30\% dieser Altersgruppe (64 Personen) nahmen keine Bildungsund Unterstützungsangebote neben der Beratung im Asylsozialdienst wahr. In den Anmerkungen der befragten Asylsozialarbeiter*innen wird darauf hingewiesen, dass neben der schwierigen psychischen Situation auch die nicht vorhandene Einbindung in familiäre Unterstützungsnetzwerke zum Abbruch von schulischen Angeboten und Sprachkursen führte. Die Bedarfe von jungen Erwachsenen mit Beeinträchtigungen, die ohne ihren Familienverbund in den Unterkünften leben, müssen insofern besonders in den Blick genommen werden. Zehn junge Frauen und 118 junge Männer mit Beeinträchtigungen im Alter von 18 bis 24 Jahren waren laut der Abfrage unbegleitet, also ohne ihre Familie, in den Unterkünften untergebracht.

Abbildung Nr. 5: Familiensituation nach Alter und Geschlecht; Quelle: Eigene Darstellung; Abfrage des Sozialreferats LH München 08/2018

Familiensituation nach Alter weibliche Personen

200

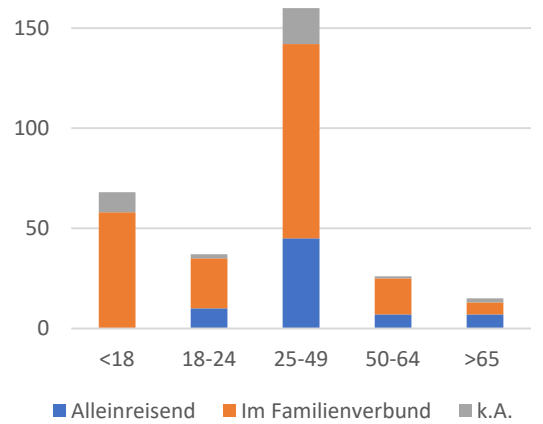

Familiensituation nach Alter männliche Personen

250

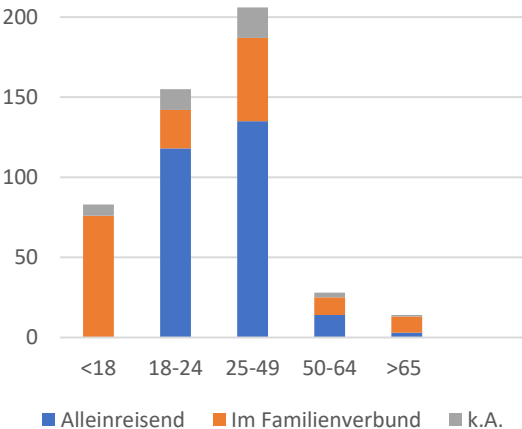

\section{Fazit und Praxisimplikationen}

Die Exploration des Feldes in München zeigt, dass zum Abfragezeitpunkt mindestens 10\% der Bewohner*innen in Münchner Unterkünften mit Be- 
hinderungen und chronische Erkrankungen lebten. Werden diese Daten von den Zahlen des ZBFS zu Menschen aus Hauptherkunftsländern in München ergänzt, so lässt sich erschließen, dass weitere Personen von barrierereichen Unterbringungs- und Lebenssituationen am Schnittpunkt von Flucht und Behinderung betroffen waren und sind. Problemlagen von Geflüchteten mit Behinderung sind heterogen und differenzieren sich, so zeigen die Ergebnisse, nach Alter, Art der Behinderung, Familiensituation und Art der Unterbringungsform. Insbesondere in AnKER-Zentren kann von einer Potenzierung der Teilhabebarrieren ausgegangen werden. Bis zur Erfüllung der von BMFSFJ und UNICEF (2018) dargestellten Mindeststandards für Geflüchtete mit Behinderungen in den Unterkünften ist noch ein langer Weg zu gehen.

Inklusive Haltungen der UN-BRK schlagen sich bislang nur mangelhaft im rechtlich restriktiven „Fluchtraum“ nieder. Der sowohl von den Disability Studies als auch in der Fluchtforschung eingeforderte Perspektivwechsel der fluiden Kategorien zeigt sich in der kommunalen Berichterstattung aufgegriffen (Landeshauptstadt München, Koordinierungsbüro zur Umsetzung der UN-Behindertenrechtskonvention, 2019; Landeshauptstadt München, Stelle für interkulturelle Arbeit, 2018). In der Praxis wird er jedoch durch die gesetzlichen Regelungen verunmöglicht, da diese konkrete Definitionen und Diagnosen für den Zugang zu Unterstützungsleistungen zum Beispiel im Bereich der Eingliederungshilfen voraussetzen. Auf der anderen Seite wird deutlich, dass selbst diese rechtlich und medizinisch zugeschriebenen Kategorien kein Garant für die Zugänglichkeit der Hilfssysteme sind. Auch die Lebenssituation von anerkannten Geflüchteten und geflüchteten Personen mit Schwerbehindertenausweis in München ist durch Exklusionserfahrungen geprägt, wie die Abfrage verdeutlicht. Besorgniserregend ist die Koppelung der Teilhaberechte mit Aspekten des rechtlichen Status und lingualer/kultureller Anpassung an die Aufnahmegesellschaft wie sie sich in den Ergebnissen der Abfragen und im derzeitigen Forschungsstand zeigt (u.a. Köbsell, 2019). Nicht nur gesetzliche Ausschlüsse verhindern die Teilhabe, auch die Bereiche der Migrationsarbeit und Behindertenhilfe haben bislang keine diversitäts- und intersektionaliätsbewußte Angebotslandschaft ausgebildet. Konzepte von Barrierefreiheit werden einseitig in Bezug auf Mobilitätsbeeinträchtigung verstanden und selbst diese zeigt sich in den Unterkünften für Geflüchtete in München nur teilweise umgesetzt und durch intransparente Prozesse und bürokratische Vielschichtigkeit behindert. Die Fachkräfte in der Migrationsarbeit und Behindertenhilfe sehen sich hohen Anforderungen gegenübergestellt. Mitarbeiter*innen in München fühlen sich aufgrund der hochkomplexen strukturellen und rechtlichen Rahmenbedingungen überfordert, wie be- 
reits in anderen Studien dargestellt (AWO Bundesverband e.V., 2017; Steiner, o.D.). Sie verfügen zum Beispiel nicht über das notwendige Wissen zu asylrechtlichen Bedingungen, welche die Lebenssituation von Geflüchteten mit Beeinträchtigungen prägen, oder zur hochkomplexen Angebotslandschaft der Behindertenhilfe. Da ein zeitnaher struktureller Wandel $\mathrm{zu}$ Regelangeboten für eine diverse und heterogene Bevölkerung nicht absehbar ist, sollte es in einem ersten Schritt mindestens zur Ausbildung von intersektional vernetzten Wissensbeständen kommen. Hierzu sollen nicht nur Wissensbestände der Behindertenhilfe und Migrationsarbeit ausgetauscht werden, sondern es muss auch neues intersektionales Wissen am Schnittpunkt generiert werden.

\section{Literaturverzeichnis}

AWO Bundesverband e.V. 2017. Beitrag des AWO Bundesverbandes zur Konsultation „Versorgungs- und Unterbringungssituation von Flüchtlingen mit Behinderungen“. https://www.awo.org/sites/default/files/2017-02/Beitrag\%20des\%20A WO\%20Bundesverbandes\%20Verb\%C3\%A4ndekonsultation\%2014.02.2017\%2 Ofinal.pdf

Bundesamt für Migration und Flüchtlinge 2020. Das Bundesamt in Zahlen 2019. Modul Asyl. Nürnberg.

Bundesministerium für Arbeit und Soziales 2016. Zweiter Teilhabebericht der Bundesregierung über die Lebenslagen von Menschen mit Beeinträchtigung. Teilhabe- Beeinträchtigung- Behinderung. Bonn.

Degener, Theresia 2016. Völkerrechtliche Grundlagen und Inhalt der UN BRK. In Degener, Theresia, Eberl, Klaus, Graumann, Sigrid, Maas, Olaf \& Schäfer, Gerhard K., hg. Menschenrecht Inklusion. 10 Jahre UN-Behindertenrechtskonvention - Bestandsaufnahme in sozialen Diensten und diakonischen Handlungsfeldern. Göttingen: Vandenhoeck et Ruprecht, 11 - 51.

Engin, Kenan 2019. Deutsche Versorgungsstrukturen im Umgang mit geflüchteten Kindern mit Behinderung im Lichte von Grundlagen des internationalen und nationalen Rechts. In M. Westphal \& G. Wansing, hg. Migration, Flucht und Behinderung: Herausforderungen für Politik, Bildung und psychosoziale Dienste. Wiesbaden: Springer VS, 103-120.

Degele, Nina \& Winker, Gabriele 2009. Intersektionalität Zur Analyse sozialer Ungleichheiten. (Sozialtheorie intro). Bielefeld: transcript.

Die Fachverbände für Menschen mit Behinderung 2019. Teilhabe von Menschen mit Behinderungen und Migrations- oder Fluchthintergrund verbessern! Forderungspapier der Fachverbände für Menschen mit Behinderung. Berlin.

Gag, Maren \& Weiser, Barbara 2017. Leitfaden zur Beratung von Menschen mit einer Behinderung im Kontext von Migration und Flucht. Passage gGmbH/Caritasverband für die Diözese Osnabrück e. V, hg. 
Goodley, Daniel 2015. Unkonventionelle Vorschläge zur Konzeptualisierung von Migration und Behinderung. In Cattacin, Sandro, Domenig, Dagmar \& Radu, Irina, hg. Vielfältig anders sein: Migration und Behinderung. Teilhabe und Verschiedenheit. Zürich: Seismo, 44-60.

Grotheer, Angela 2017. Eine Annäherung an die Lebensbedingungen von Menschen im Asyl und in der Duldung am Beispiel von Hamburg, hg.Universität Hamburg.

Grotheer, Angela \& Schroeder, Joachim 2019. Unterbringung von Geflüchteten mit einer Behinderung. In M. Westphal \& G. Wansing, hg. Migration, Flucht und Behinderung: Herausforderungen für Politik, Bildung und psychosoziale Dienste. Wiesbaden: Springer VS, 81-101.

Handicap International 2019. Dokumentation des dritten Treffens des bundesweiten Netzwerkes Flucht, Migration und Behinderung. https://handicap-internatio nal.de/sn_uploads/de/document/Dokumentation_des_dritten_bundesweiten_N etzwerktreffenens_Flucht_Migration_und_Behinderung_Koln_11_und_12 _06_2019.pdf

Hess, Sabine, Kasparek, Bernd, Kron, Stefanie, Rodatz, Mathias, Schwertl, Maria \& Sontowski, Simon 2017. Der lange Sommer der Migration. Krise, Rekonstitution und ungewisse Zukunft des europäischen Grenzregimes. In S. Hess u. a., hg. Hess, Sabine; Kasparek, Bernd; Kron, Stefanie; Rodatz, Mathias; Schwertl, Maria; Sontowski, Simon. Der lange Sommer der Migration: Assoziation a, 6-24.

Hirschberg, Marianne \& Köbsell, Swantje 2016. Rundbegriffe und Grundlagen: Disability Studies, Diversity und Inklusion. In Biewer, Gottfried; Hedderich, Ingeborg; Hollenweger, Judith; Markowetz, Reinhard, hg. Handbuch Inklusion und Sonderpädagogik, Bad Heilbrunn: Klinkhardt, 555-568.

Kleist, J. Olaf 2019. Flucht- und Flüchtlingsforschung in Deutschland: Die Etablierung eines Forschungsfeldes. In B. Behrensen \& M. Westphal, hg. Fluchtmigrationsforschung im Aufbruch: Methodologische und methodische Reflexionen. Wiesbaden: Springer, 11-24.

Köbsell, Swantje 2019. „Disabled asylum seekers? ... They don't really exist.“ Zur Unsichtbarkeit behinderter Flüchtlinge im Hilfesystem und behindertenpolitischer Diskurs. In M. Westphal \& G. Wansing, hg. Migration, Flucht und Behinderung: Herausforderungen für Politik, Bildung und psychosoziale Dienste. Wiesbaden: Springer VS, 63-80.

Korntheuer, Annette, Afeworki Abay, Robel \& Westphal, Manuela 2021. Forschen in den Feldern von Flucht und Behinderung: Ein Vergleich von forschungsethischen Herausforderungen und notwendigen forschungspraktischen Rahmenbedingungen. In Franz, Julia \& Unterkoffer, Ursula, hg. Forschungsethik in der Sozialen Arbeit: Prinzipien und Erfahrungen. Leverkusen: Verlag Barbara Budrich, 229-242.

Korntheuer, Annette 2020. Intersektionale Ausschlüsse am Schnittpunkt Flucht und Behinderung. Erste Analysen in der Landeshauptstadt. Zeitschrift für Inklusion, (3). 
Korntheuer, Annette \& Hergenröther, Sarah 2020. How Cities responded: Answers and Challenges in the City of Munich. In Korntheuer, Annette, Maehler, Debora, Pritchard, Paul \& Wilkinson, Lori hg. Refugees in Canada and Germany: Responses and policy and practice (GESIS Working Papers). Köln, 25-44.

Kruse, Jan 2015. Qualitative Interviewforschung. Ein integrativer Ansatz. Grundlagentexte Methoden. Weinheim; Basel: Beltz Juventa.

Landeshauptstadt München 2014. 1.Aktionsplan zur Umsetzung der UN-Behindertenrechtskonvention. Koordinierungsbüro zur Umsetzung der UN-Behindertenrechtskonvention. München.

Landeshauptstadt München 2019. 2.Aktionsplan zur Umsetzung der UN-Behindertenrechtskonvention. Koordinierungsbüro zur Umsetzung der UN-Behindertenrechtskonvention. München.

Landeshauptstadt München 2018. Münchner Gesamtplan zur Integration von Flüchtlingen. Stelle für interkulturelle Arbeit. München.

Landeshauptstadt München 2020. Münchner Gesamtplan zur Integration von Flüchtlingen. Abschlussbericht und Perspektiven. Stelle für interkulturelle Arbeit. München.

Leisering, Britta 2018. Geflüchtete Menschen mit Behinderungen: Handlungsnotwendigkeiten für eine bedarfsgerechte Aufnahme in Deutschland. Deutsches Institut für Menschenrechte, hg. Berlin.

Intersektionale Ausschlüsse am Schnittpunkt Flucht und Behinderung. Erste Analysen in der Landeshauptstadt. Zeitschrift für Inklusion, (3).

Otten, Matthias 2019. Partizipative Forschung zur Teilhabe von geflüchteten Menschen mit Behinderung. In V. Klomann u. a., hg. Forschung im Kontext von Bildung und Migration. Wiesbaden: Springer, 181-194.

Otten, Matthias, Schahrzad, Farrokhzad \& Zuhr Anna 2017. Flucht und Behinderung als Schnittstellenaufgabe der Sozialen Arbeit. Gemeinsam Leben. Zeitschrift für Inklusion, 25(4), 197-206.

Pearce, Emma 2015. „Ask us what we need“: Operationalizing Guidance on Disability inclusion in Refugee and Displaced Persons Programs. Disability and the Global South, 2(1), 460-478.

Rathgeb, Kerstin 2015. Ein Versuch, Intersektionalität und Interdependenz für die Behindertenarbeit auf die Füsse zu stellen. In Cattacin, Sandro, Domenig, Dagmar, Radu, Irina, hg. Vielfältig anders sein: Migration und Behinderung, Teilhabe und Verschiedenheit. Zürich: Seismo, 88 - 103.

Scherr, Albert \& Scherschel, Karin 2019. Wer ist ein Flüchtling? Grundlagen einer Soziologie der Zwangsmigration: Göttingen: Vandenhoeck \& Ruprecht.

Schülle, Mirjam 2019. Medizinische Versorgung für Menschen mit Behinderungen, die Leistungen nach dem Asylbewerberleistungsgesetz erhalten. In Wansing, Gudrun \& Westphal, Manuela, hg. Migration, Flucht und Behinderung. Herausforderungen für Politik, Bildung und psychosoziale Dienste. Wiesbaden: Springer VS, $145-165$. 
Schwalgin, Susanne \& Wank, Ricarda 2017. Stellungnahme zum Thema Flüchtlinge mit Behinderung für das Deutsche Institut für Menschenrechte, hg. Handicap International. https://www.netzwerk-iq.de/fileadmin/Redaktion/Downloads /Fachstelle_Einwanderung/TT8/Fachstelle_Einwanderung_8.Thementage_Stellu ngnahme_DIMR_Handicap_International_2017.pdf

Statistisches Bundesamt 2019. Bevölkerung und Erwerbstätigkeit Schutzsuchende Ergebnisse des Ausländerzentralregisters, 1.Fachserie, 2.4 Reihe.

Steiner, Max (o.D.). Umfrage zur Versorgungslage geflüchteter Menschen mit Behinderung in Deutschland innerhalb des deutschen Caritasverbandes. Deutscher Caritasverband e.V., hg.

Walgenbach, Katharina 2016. Intersektionalitätsforschung. In Biewer, Gottfried, Hedderich, Ingeborg, Hollenweger, Judith \& Markowetz, Reinhard, hg. Handbuch Inklusion und Sonderpädagogik. Bad Heilbrunn: Klinkhardt, 650 - 655.

Wansing, Gudrun \& Westphal, Manuela 2019. Schnittstelle von Migration und Behinderung in Bewegung. In Westphal, Manuela \& Wansing, Gudrun, hg. Migration, Flucht und Behinderung. Herausforderungen für Politik, Bildung und psychosoziale Dienste. Wiesbaden: Springer VS., 4-23.

Yeo, Rebecca-Amani 2015. "Disabled asylum seekers? ... They don't really exist": The marginalisation of disabled asylum seekers and why it matters. Disability and the Global South, 2(1), 523-550. 
